\title{
Das soziale Europa im Räderwerk des Steuerwettbewerbs
}

Im Steuerwettbewerb kommt das soziale Europa unter die Räder. Durch ihr Festhalten an einer vermeintlichen Steuersouveränität, die sie durch die ökonomische Integration in Wirklichkeit längst verlorenen haben, unterminieren die Mitgliedstaaten gegenseitig ihre (unterschiedlichen) Sozialstaatsmodelle. Der Beitrag stellt dar, wie der europäische Steuerwettbewerb funktioniert, welche Konsequenzen er hat und wieso die bisherigen Bemühungen zu seiner Begrenzung nicht ausreichen. Abschließend wird skizziert, wie die Mitgliedstaaten durch eine gemeinsame Politik der Europäischen Union (EU) ihre Gestaltungsfähigkeit zurückgewinnen können. Dies ist die Voraussetzung dafür, dass sie ein soziales Europa schaffen und ihre Sozialmodelle erfolgreich umsetzen können.

THOMAS RIXEN

\section{Einleitung}

Die Bekämpfung des schädlichen Steuerwettbewerbs und der internationalen Steuerflucht hat derzeit eine hohe politische Priorität. Die beiden wichtigsten Gründe dafür sind einmal die Finanz-, Euro- und Staatsschuldenkrise, in deren Folge viele Staaten sich unerschlossene Einnahmen nicht weiter entgehen lassen wollen, um ihre überschuldeten Haushalte zu sanieren. Zum anderen werden die politischen Bemühungen angefacht durch die Aufdeckung prominenter Steuerskandale von Uli Hoeneß über Apple, Starbucks bis Offshore-Leaks, Luxleaks und SwissLeaks, die bei der großen Mehrzahl ehrlicher Steuerzahler und Wähler Empörung auslösen.

In diesem Beitrag argumentiere ich, dass ein Ende des schädlichen Steuerwettbewerbs und eine gelingende steuerpolitische Kooperation in der EU nicht nur als Reaktion auf diese aktuellen Ereignisse notwendig ist, sondern eine wesentliche Voraussetzung für die Realisierung eines sozialen Europa ist. Der Steuerwettbewerb in seiner aktuellen Form sorgt nicht nur dafür, dass den Staaten Steuereinnahmen zur Verfolgung sozialer Ziele entgehen, sondern er führt auch dazu, dass das wichtigste umverteilungspolitische Instrument, nämlich die progressive direkte Besteuerung, stumpf wird. Wie zuletzt erneut von Piketty (2014) gezeigt, sind die steuerpolitischen Reformen der letzten drei Dekaden, zu denen sich die Regierungen maßgeblich unter dem
Druck des Steuerwettbewerbs gezwungen sahen, ein Hauptgrund für die Zunahme der Ungleichheit. Eine Umkehr dieses Trends wird nur dann gelingen, wenn die Regierungen in der Zukunft eine andere Politik verfolgen. Damit dies möglich wird, ist es notwendig, eine gemeinsame europäische Steuerpolitik zu entwickeln. Die Kernthese dieses Beitrags liegt in der nur scheinbar paradoxen Aussage, dass die Mitgliedstaaten ihre De-jure-Souveränität teilen müssen, um ihre tatsächliche (De-facto-)Souveränität zurückzuerobern. Nur wenn es gelingt, dass man sich auf europäische Standards und einige gemeinsame Regeln der Besteuerung einigt, werden die Mitgliedstaaten ihre steuer- und damit verteilungspolitische Gestaltungsfähigkeit zurückerlangen, die sie im Zuge der ökonomischen Integration an den Steuerwettbewerb verloren haben. Damit schafft die Steuerkooperation überhaupt erst die Voraussetzungen für ein sozial gerechtes Europa. Das soziale Europa zeichnet sich durch die gemeinsame Überzeugung aus, dass es eines starken sozialen Ausgleichs und einer Einbettung des Marktes bedarf, aber auch durch national unterschiedliche Traditionen, Institutionen und Sozialstaatsmodelle zur Verwirklichung dieser Überzeugung.

In Abschnitt 2 erläutere ich zunächst, wie schädlicher Steuerwettbewerb in Europa funktioniert. Dabei unterscheide ich zwischen verschiedenen Arten des Steuerwettbewerbs (realer vs. virtueller und generalisierter vs. präferentieller Wettbewerb). In Abschnitt 3 zeige ich, welche negativen Konsequenzen der Steuerwettbewerb für eine sozial 
gerechte Politik im Allgemeinen und die Entwicklung der Einkommensungleichheiten im Besonderen hat. Anschließend stelle ich dar, welche Maßnahmen gegen schädlichen Steuerwettbewerb in der EU bisher unternommen wurden (4). Trotz des häufig geäußerten Bekenntnisses zu einem sozialen Europa ist es den Regierungen bisher kaum gelungen, den schädlichen Steuerwettbewerb effektiv zu regulieren. Zwar hat man bei der administrativen Kooperation zwischen Steuerbehörden und der Herstellung von Transparenz wichtige Fortschritte erzielt. Allerdings gibt es keine Bemühungen, den generalisierten Steuerwettbewerb, also die Absenkung der Steuersätze um Investitionen anzulocken, zu begrenzen. Dies würde eine zumindest teilweise Harmonisierung zentraler Parameter der nationalen Steuersysteme, insbesondere der Bemessungsgrundlage und der Steuersätze, erforderlich machen. Eine solche parametrische Kooperation scheitert bisher aber an den Interessen der (kleinen) Mitgliedstaaten, die vom Steuerwettbewerb profitieren. In Abschnitt 5 werden eine solche parametrische Kooperation in Form einer gemeinsamen konsolidierten Körperschaftsteuerbemessungsgrundlage (GKKB) mit Mindeststeuersatz vorgeschlagen und deren Umsetzungschancen diskutiert. Abschließend werden politische Implikationen benannt (6).

\section{Wie funktioniert der Steuerwettbewerb?}

Steuerwettbewerb wird im Wesentlichen um mobiles Kapital geführt. Wettbewerb um den weniger mobilen Produktionsfaktor Arbeit findet kaum statt. ${ }^{1}$ Beim Wettbewerb um Kapital kann man zwischen realem und virtuellem Steuerwettbewerb unterscheiden.

Im Wettbewerb um ausländische Direktinvestitionen konkurrieren die Staaten um reale ökonomische Aktivität. Sie versuchen, die Standortentscheidungen international agierender Konzerne mittels Steuern zu beeinflussen. Diese realen Investitionsentscheidungen hängen zwar vor allem von nicht-steuerlichen Faktoren ab ((Aus-)Bildungsniveau, Arbeitskosten, Qualität der Infrastruktur vor Ort etc.), aber Steuern können ebenfalls eine Rolle spielen. Hinsichtlich der Frage, ob ein Einfluss der Steuern auf diese realen ökonomischen Entscheidungen erkennbar ist, kommen die vorhandenen empirischen Studien zu unterschiedlichen Ergebnissen. In ihrer Metaanalyse, die alle verfügbaren Studien zu dem Thema umfasst, arbeiten De Mooij und Ederveen (2008) heraus, dass der Einfluss erkennbar, aber nicht sehr groß ist.

Neben dem realen gibt es aber auch einen virtuellen Steuerwettbewerb, wobei sich zwei Unterkategorien unterscheiden lassen. Erstens führen die Regierungen, ebenfalls im Bereich der Unternehmensbesteuerung, Wettbewerb um sogenannte Buchgewinne (paper profits). Dabei geht es um die legale Steuervermeidung von multinationalen Unter- nehmen (MNU). Möglich wird die Verlagerung von Buchgewinnen, weil das internationale und das europäische Steuerrecht auf dem Prinzip des sogenannten „seperate entitity accounting" beruht, das heißt, die verschiedenen Teile eines MNU werden strikt national getrennt besteuert, so als wären sie vollkommen unabhängige Einheiten. Aufgrund dieses Prinzips können MNUs beispielsweise Finanzierungsgesellschaften einsetzen, die ihren Sitz in Niedrigsteuerländern bzw. Steueroasen haben. Gewinne können dann dorthin verlagert werden, um die konzernweite Steuerlast zu minimieren. Dies kann zum Beispiel durch einen sehr hohen Fremdfinanzierungsanteil geschehen oder durch die Manipulation firmeninterner Verrechnungspreise.

Eine andere Strategie ist die Auslagerung von Lizenzrechten an Tochterunternehmen in Niedrigsteuerländer. Die Firmenteile in Normalsteuerländern müssen dann möglichst hohe Lizenzgebühren für die Nutzung beispielsweise von Software oder Markenrechten abführen, sodass dort (gewinnund steuermindernde) Kosten entstehen, während der zu versteuernde Gewinn im Niedrigsteuerland realisiert wird. Letzteres hat z. B. zu den extrem niedrigen Steuerzahlungen von Firmen wie Apple, Google und Starbucks geführt, die in Medien und Öffentlichkeit für Aufsehen gesorgt haben. Tatsächlich kommen alle empirischen Untersuchungen zu dem Ergebnis, dass die deklarierten Unternehmensgewinne sehr elastisch auf Länderunterschiede im entsprechenden Steuersatz reagieren (siehe z. B. De Mooij/Ederveen 2008). Der relevante Faktor für die Entscheidung, Buchgewinne in einem Land zu verbuchen, ist der Spitzensatz der Körperschaftsteuer. Wie in Abbildung 1 zu erkennen ist, ist dieser in der EU infolge des Steuerwettbewerbs deutlich gesunken.

Zweitens konkurrieren die Staaten auch um Portfoliokapital natürlicher Personen. Auf die Spitze getrieben wird dieser Wettbewerb von der Gruppe von Staaten bzw. unabhängigen Gebieten, die sich als Steueroasen positioniert haben. Ihr Geschäftsmodell basiert auf extrem niedrigen oder gar keinen Steuern und einem strikten Bank- und Steuergeheimnis. So können sie zur illegalen Steuerhinterziehung durch Privatpersonen genutzt werden. Dazu werden häufig Stiftungen (Trusts), besondere Fonds oder andere spezielle Rechtskonstruktionen angeboten, die es den Investoren erlauben, anonym zu bleiben. Zwar sind die Steuerzahler nach dem Gesetz verpflichtet, die entsprechenden Gelder dem Finanzamt zu melden, was sie aber bisher nahezu gefahrlos unterlassen konnten, da die Heimatländer aufgrund der Verschleierung nichts von dem im Ausland angelegten Geld erfahren. International tätige Banken bieten ihren wohlhabenden Kunden maßgeschneiderte Offshore-Strukturen an, bei denen das Geld oft durch mehrere solcher Geschäftseinheiten in verschiedene Oasen fließt,

\footnotetext{
1 Was es allerdings gibt, ist die Konkurrenz um den Wohnsitz von einigen Ultrareichen, denen besondere Steuervergünstigen, z. B. in Großbritannien oder in einigen Schweizer Kantonen, angeboten werden.
} 
um eine mehrfache Verschleierung der Besitzverhältnisse zu erreichen (vgl. z. B. Palan et al. 2010). Es wird geschätzt, dass weltweit ca. $8 \%$ des privaten Finanzvermögens in Steueroasen angelegt sind, dies entspricht $5.800 \mathrm{Mrd}$ €, während es in Europa sogar 12 \% des hiesigen Vermögens sind (Zucman 2014, S. 18, 49).

Sowohl den realen wie auch den virtuellen Steuerwettbewerb gibt es in zwei Varianten. Zum einen in generalisierter Form, die vorliegt, wenn die (günstigen) steuerlichen Regelungen für alle Steuerzahler, also In- wie Ausländer, gleichermaßen gelten. Außerdem gibt es auch einen Wettbewerb mittels präferentieller Regime. Dabei werden die günstigen steuerlichen Regelungen nur ausländischen Investoren angeboten, gelten aber für Inländer nicht, oder es werden nur bestimmte Arten von Investitionen (beispielsweise besonders mobile) steuerlich bevorzugt, während andere normal besteuert werden. Solche präferentiellen Regime haben für die Regierungen den Vorteil, dass man so Investitionen anlocken kann, übermäßige Steuerausfälle bei den heimischen Investitionen aber verhindert.

Wichtig ist schließlich, dass der virtuelle und reale Steuerwettbewerb nicht unabhängig voneinander sind. Wenn die Unternehmen nicht mehr mittels Gewinnverlagerung Steuervorteile realisieren könnten, dann würde man theoretisch erwarten, dass ihre Bereitschaft steigt, trotz der damit verbundenen Kosten reale Produktionsstandorte zu verlagern. Mit anderen Worten: Wenn man den virtuellen Steuerwettbewerb unterbinden würde, würde sich die Sensitivität realer Investitionsentscheidungen für Steuervorteile erhöhen. Der reale Steuerwettbewerb würde sich verschärfen. Wie stark dieser Effekt wäre, ist eine empirische Frage, die sich nicht mit Sicherheit beantworten lässt (Fuest 2008, S. 732f.).

\section{Die negativen Konsequenzen des Steuerwettbewerbs für ein soziales Europa}

Viele positive Beurteilungen des Steuerwettbewerbs beruhen implizit oder explizit auf der Vorstellung eines realen Wettbewerbs um Steuerzahler und produktive Faktoren (Tiebout 1956). Die Vorstellung ist, dass es zu einem effizienten Allokationsergebnis kommt, da sich wie auf einem Markt für Privatgüter die Steuern als Preise für öffentliche Güter, die die Steuerzahler durch Zuzug einkaufen, bilden. Ganz unabhängig davon, dass ökonomische Effizienz angesichts der Eigenschaften öffentlicher Güter in einem Wettbewerbsprozess nicht erreicht werden kann (Sinn 1997), hat der vorherige Abschnitt gezeigt, dass der real existierende Steuerwettbewerb vorwiegend ein virtueller ist. Die Unternehmen oder Individuen bleiben mit ihren wesentlichen Produktions- und Verwaltungsstandorten bzw. dem tatsächlichen Wohnsitz in den Normalsteuerländern und ver- lagern lediglich ihre steuerpflichtigen Einkünfte oder Vermögen in Niedrigsteuerländer. Die reale wirtschaftliche Aktivität wird getrennt von der steuerlichen Belastung. Im Ergebnis nutzen sie so die gute Infrastruktur und andere öffentliche Güter in den Ländern, in denen sie produzieren oder leben, ohne sich im eigentlich vorgesehenen Umfang an deren Finanzierung zu beteiligen.

Dies zeigt, dass die Vorstellung einer fiskalischen Äquivalenz - man zahlt Steuern als Preis für die öffentliche Leistung - durch den real existierenden Steuerwettbewerb unterminiert wird. Man hat es nicht mit einem funktionierenden Wettbewerb zu tun, sondern mit Trittbrettfahrerverhalten bzw. Schwarzfahrerei. Darin liegt eine offensichtliche Ungerechtigkeit, da die Steuerzahler mit Zugriff auf Möglichkeiten der internationalen Steuerplanung und -hinterziehung gegenüber den anderen bevorzugt werden. Gleiches gilt natürlich auch für den präferentiellen Steuerwettbewerb, auch hier wird eine Gruppe, nämlich die der ausländischen Investoren, bevorzugt.

Dieses Verdikt über die Ungerechtigkeit des Steuerwettbewerbs lässt sich mit empirischen Belegen zu dessen (Um-) Verteilungswirkung sowohl zwischen als auch innerhalb von Staaten unterfüttern. In einer ersten Annäherung kann man sagen, dass die großen, wirtschaftlich starken

\section{ABB. 1}

\section{Die Entwicklung wichtiger Steuersätze in der EU-28*, 1995 - 2014}

\section{Angaben in Prozent}

- Spitzensatz Einkommenssteuer

— Spitzensatz Körperschaftssteuer

- - = EATR** (EU-27)

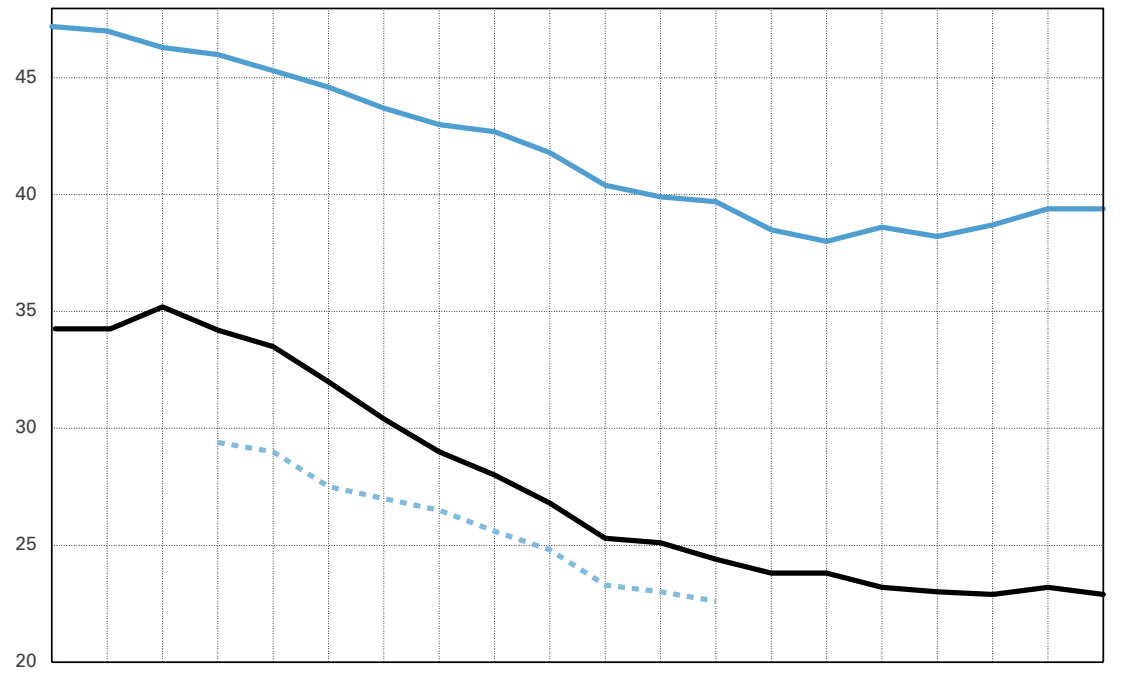

19951996199719981999200020012002200320042005200620072008200920102011201220132014

*Es sind alle 28 Mitgliedsländer der EU enthalten, auch diejenigen, die erst nach 1995 Mitglied wurden. Lediglich die Daten für Bulgarien und Rumänien sind erst ab dem Jahr 2000 bzw. 2001 berücksichtigt. „Die „effective average tax rate (EATR)“ gibt an, wie groß die durchschnittliche Belastung einer Investition ist; es ist der relevante Indikator für eine diskrete Investitionsentscheidung (ob man überhaupt an einem Standort eine Betriebsstätte eröffnet).

Quelle: Elschner/Vanborren (2009); Eurostat $(2008,2014)$. Berechnungen und Darstellung des Autors. 
Länder durch den Steuerwettbewerb verlieren, während Steueroasen, meist kleine Staaten, profitieren, da sie erfolgreich Buchgewinne und insbesondere Portfolio-Kapital anziehen können. Auf der Basis seiner Schätzungen über das insgesamt in Steueroasen liegende Vermögen errechnet Zucman (2014, S. 58), dass den großen Staaten jährlich Steuereinnahmen in Höhe von $130 \mathrm{Mrd}$ € entgehen.

Nun könnte man fragen, ob denn nicht die durchaus positive Kehrseite dieser Medaille sei, dass kleine Länder als Steueroasen eine erfolgreiche Wachstumsstrategie verfolgen können und es so zu einem Abbau der zwischenstaatlichen Unterschiede kommt. Tatsächlich sind ja in der EU in der Vergangenheit Länder wie Irland und Luxemburg, die sich erfolgreich mit niedrigen Körperschaftsteuern und maßgeschneiderten Steuervereinbarungen als Buchungszentren für die Papiergewinne multinationaler Unternehmen positioniert haben, sowie das Finanzzentrum Zypern als erfolgreiche Modelle angepriesen worden. Allerdings hat sich insbesondere in Irland und Zypern in der Finanzkrise gezeigt, dass es sich nicht um ein nachhaltiges Wachstum handelt. ${ }^{2} \mathrm{Im}$ Gegenteil hat die mit der Steueroasenstrategie einhergehende Finanzialisierung der Volkswirtschaften zu deren Beinahekollaps geführt und sie volkswirtschaftlich weit zurückgeworfen (siehe auch den Beitrag von Nölke in diesem Heft). So bleibt letztlich festzuhalten, dass der Steuerwettbewerb für die großen Staaten in Europa mit großen Kosten bzw. Verlusten verbunden ist, während die Steuerparadiese davon ebenfalls nicht nachhaltig profitieren.

\subsection{Die Auswirkungen auf die Steuereinnahmen}

Die Auffassung, dass der Steuerwettbewerb keine Gefahr für das Überleben ausgebauter Wohlfahrtsstaaten und deren Fähigkeit zur Einkommensumverteilung ist, wie sie in den 1990er und frühen 2000er Jahren in einem Teil der Literatur vertreten wurde, muss revidiert werden (näher hierzu und zum Folgenden: Rixen 2014). Diese Beiträge stützen sich insbesondere auf die Tatsache, dass die aggregierten Steuereinnahmen aus Unternehmensgewinnen und Kapitaleinkommen konstant geblieben sind. Tatsächlich hätten aber angesichts der höheren Privatvermögen und der gestiegenen Profitabilität der Unternehmen die Einnahmen aus der Kapitaleinkommen- und Körperschaftsteuer deutlich steigen müssen (vgl. z. B. Clausing 2016). Die Tatsache, dass sie lediglich konstant geblieben sind, ist ein Indiz dafür, dass der Steuerwettbewerb die Einnahmekapazität der Staaten geschwächt hat (siehe auch Godar et al. 2015).

Dies zeigt sich, wenn man nicht die tatsächlich erzielten Steuerquoten bzw. Steuersätze (Ex-post-Sätze), sondern die Entwicklung der Ex-ante-Sätze analysiert, die angeben, wie hoch die tatsächliche steuerliche Belastung für ein typisches Investitionsobjekt nach Lage der Gesetze ausfallen würde. Diese Sätze geben also an, wie hoch die von der Politik erwünschte effektive Steuerbelastung ist. Wie in Abbildung 1 zu erkennen ist, sind diese Sätze im Durchschnitt der EU-
Mitgliedsländer gesenkt worden, was dafür spricht, dass sich die Staaten in einem Steuerwettbewerb wähnten. Gleiches gilt für die nominalen Sätze der Körperschaftsteuer, die für den Wettbewerb um Buchgewinne relevant sind.

\subsection{Die Auswirkungen auf soziale Ungleichheit}

Aber nicht nur durch ausbleibende Einnahmen schwächt der Steuerwettbewerb die Aussicht auf ein soziales Europa. Er führt auch zu einer Zunahme der sozialen Ungleichheit, da er eine Verschiebung der Steuerlasten verursacht:

Erstens hat die Art, wie die Unternehmenssteuern gesenkt wurden, Konsequenzen für die Verteilung der Steuerlast auf verschiedene Arten von Kapital. In fast allen EULändern hat man die Bemessungsgrundlagen erheblich erweitert, um die Absenkung der nominalen Sätze zumindest teilweise gegenzufinanzieren. Die Folge ist, dass große MNUs von der Absenkung der nominalen Körperschaftsteuersätze profitieren, von der Verbreiterung der Bemessungsgrundlage aber nicht erfasst werden, weil sie die Gewinne aus dem Land verlagern können. National aufgestellte klein- und mittelständische Unternehmen dagegen werden von der Verbreiterung der Bemessungsgrundlage voll erfasst. Mit anderen Worten, international mobiles Kapital wird entlastet, während national gebundenes Kapital stärker belastet wird.

Zweitens wurde die Steuerlast von Kapital zu Arbeit und Konsum verlagert. Der relative Durchschnittssteuersatz für Kapital im Vergleich zu Arbeit und Konsum sinkt in den großen Industrieländern (Winner 2005). Dies zeigt sich u.a. in gestiegenen Mehrwertsteuersätzen, die insbesondere ärmere Haushalte, die einen größeren Teil ihres Einkommens für den Konsum ausgeben, belasten.

Drittens macht es der Steuerwettbewerb den Staaten deutlich schwieriger, ein progressives, auf Umverteilung zielendes Einkommensteuersystem aufrechtzuerhalten. Die Ursache dafür liegt in der sogenannten „Schutzfunktion" (backstop function) der Körperschaftsteuer für die Einkommensteuer. Damit es den Steuerzahlern unmöglich gemacht wird, ihr persönliches Einkommen in Körperschaften zu „verstecken“, um in den Genuss von niedrigeren Steuersätzen zu gelangen, sollten der Spitzensatz der persönlichen Einkommensteuer und der Unternehmenssteuersatz möglichst eng beieinander liegen. Durch die Absenkung der Unternehmenssteuersätze ist deshalb ein Druck auf die Sätze der persönlichen Einkommensteuer

2 Die Ursache dafür ist, dass die meisten Gelder nicht in die Realwirtschaft der Steuerparadiese investiert werden, sondern dort lediglich die Verwaltung von Anlagedepots, Stiftungen und Trusts stattfindet. Der Wachstumseffekt in den Steuerparadiesen liegt also allein im höheren Geschäftsaufkommen im Bereich der Vermögensverwaltung und damit in der Finanzbranche. 
entstanden (Ganghof/Genschel 2008), die im EU-Durchschnitt deutlich gesenkt worden sind (Abbildung 1). Wie Piketty (2014, Kap. 14) gezeigt hat, ist der Zusammenhang zwischen gesunkenen Spitzensätzen der Einkommensteuer und zunehmender Ungleichheit sehr stark. Die Staaten, in denen die Spitzensteuersätze der Einkommensteuer seit den 1980er Jahren am meisten gesenkt wurden, sind auch diejenigen, in denen der Anteil der obersten $10 \%$ der Einkommensbezieher am Volkseinkommen am stärksten gestiegen ist.

Die beschriebenen Mechanismen und Konsequenzen des Steuerwettbewerbs gelten sowohl global als auch in der EU. Allerdings sorgen institutionelle Grenzen dafür, dass der Steuerwettbewerb in der EU besonders scharf geführt wird. Zum einen schafft der gemeinsame Markt besonders weitgehende Freiheiten für den Kapitalverkehr (starke negative Integration), zum anderen ist die EU nur in begrenztem Maße in der Lage, regulierend in den Steuerwettbewerb einzugreifen (schwache positive Integration).

Die EU verfügt nicht über ein eigenes Besteuerungsrecht, sondern dieses liegt bei den Nationalstaaten. Nichtsdestoweniger kann die EU sekundäres Recht im Steuerbereich verabschieden und damit den Mitgliedstaaten bei der Ausübung ihres Besteuerungsrechts Grenzen auferlegen (Genschel/Jachtenfuchs 2011). Allerdings ist dafür Einstimmigkeit im Rat erforderlich. Es zeigt sich aber, dass die EU vorrangig im Bereich der indirekten Steuern (v.a. Verbrauchsteuern und allgemeine Mehrwertsteuer) tätig geworden ist. Beim Normalsatz der Mehrwertsteuer gilt ein Mindeststeuersatz von $15 \%$. Der durchschnittliche Normalsatz in der EU ist von 19,3\% im Jahr 2000 auf 21,5\% (2014) angestiegen (Eurostat 2014, S. 25) - nicht zuletzt, weil die Mehrwertsteuer eine sichere Einnahmequelle darstellt, die steuerwettbewerbsresistent ist.

Demgegenüber gab es bei den direkten Steuern, die dem Steuerwettbewerb unterliegen, zwar immer wieder Initiativen zur Regulierung. Diese sind aber meist nicht erfolgreich gewesen, weil die Mitgliedsländer, die vom Steuerwettbewerb profitieren (insbesondere kleine Staaten wie Irland und Luxemburg, aber auch das Vereinigte Königreich), die von den großen Staaten, die im Steuerwettbewerb verlieren, angestoßenen Initiativen blockierten bzw. verwässerten (Wasserfallen 2014). Schließlich kommt hinzu, dass der Europäische Gerichtshof verschiedene Entscheidungen zugunsten von Steuerzahlern getroffen hat, die im Namen der Kapitalverkehrsfreiheit einen stark liberalisierenden Effekt hatten und den Steuerwettbewerb in der EU weiter angefacht haben. Im Ergebnis fällt insbesondere in der Unternehmensbesteuerung die Senkung der Steuersätze deutlich stärker aus als in anderen Industrieländern (Genschel et al. 2011). Insgesamt zeigt sich also auch in der Steuerpolitik, dass die EU mit ihrer Politik der Marktschaffung und Liberalisierung dazu beigetragen hat, die Ungleichheiten innerhalb der Mitgliedstaaten zu verschärfen (siehe Beckfield und Nölke in diesem Heft).

\section{Die bisherige Politik gegen schädlichen Steuerwettbewerb}

Im Folgenden bespreche ich die wichtigsten Initiativen gegen den Steuerwettbewerb in der EU. Dabei wird gezeigt, dass lediglich der Kampf gegen präferentiellen und virtuellen Wettbewerb aufgenommen wurde, der mittels verstärkter administrativer Kooperation zwischen den Mitgliedstaaten geführt wird. Demgegenüber fehlen Maßnahmen der parametrischen Koordination, die notwendig wären, um den generalisierten Wettbewerb zu begrenzen, der umso stärker werden wird, wenn der virtuelle Wettbewerb beschränkt wird.

\subsection{Der Kampf gegen Steuerhinterziehung}

Am weitesten entwickelt sind die Maßnahmen gegen internationale Steuerhinterziehung. Die EU-Zinsrichtlinie, die nach jahrzehntelangen Bemühungen 2003 verabschiedet wurde und 2005 in Kraft trat, sieht vor, dass Informationen zwischen den EU-Mitgliedern und einigen Nichtmitgliedern (insbesondere umliegenden Steueroasen) über die Zinseinkommen ausländischer Anleger ausgetauscht werden. Dieser Informationsaustausch erfolgt automatisch, das heißt die Steuerbehörden melden Informationen zu den Konten aller ausländischen Anleger an deren Heimatländer, ohne dass diese eine Anfrage stellen müssen. Die Zinsrichtlinie galt vielen in der Zivilgesellschaft und Politik, die zu Recht einen umfassenden automatischen Informationsaustausch als effektive Lösung gegen Steuerhinterziehung sehen, als Modell für eine weitergehende globale Steuerkooperation (Rixen 2013).

Allerdings wies die Zinsrichtlinie in ihrer ursprünglichen Fassung erhebliche Schwachstellen auf. Zum einen konnten einige Steueroasen, darunter die EU-Mitglieder Österreich und Luxemburg, nur dadurch zur Unterzeichnung bewegt werden, dass man ihnen anbot, statt des automatischen Informationstausches eine Quellenabzugsteuer einzuführen. Drei Viertel der Einnahmen flossen anonym, also ohne Nennung der Anleger, an die Heimatstaaten. Der Steuersatz betrug zunächst 15, dann 20 und seit $201135 \%$. Zweitens war der Einkommensbegriff sehr eng, nur Zinseinkommen waren abgedeckt. So konnten Steuerhinterzieher die Richtlinie umgehen, indem sie statt in zinstragende Papiere in Aktien investierten. Oder sie gründeten eine Stiftung, die als Unternehmen ebenfalls nicht unter die Zinsrichtlinie fiel. Empirisch zeigt sich, dass diese Umgehungsmöglichkeiten tatsächlich ausgiebig genutzt wurden (Rixen/ Schwarz 2012), was einer breiten Öffentlichkeit durch die Zumwinkel-Affäre und Offshore-Leaks bekannt wurde. Außerdem gilt die Zinsrichtlinie im Wesentlichen nur EU-weit und nicht global, man konnte sie also durch Anlagen in Drittstaaten umgehen.

Auch wenn die EU Vorreiterin bezüglich des automatischen Informationsaustausches war, so wurde sie doch 
zwischenzeitlich von den globalen Entwicklungen überholt. Die USA verabschiedeten den sog. Foreign Account Taxpayer Compliance Act (FATCA), der ausländische Banken bzw. Regierungen dazu verpflichtet, automatisch Daten über ihre US-amerikanischen Kunden an die US-Finanzbehörden zu liefern. ${ }^{3}$ FATCA hat wesentliche Veränderungen in der internationalen Steuerpolitik angestoßen. Zum einen hat es den Bemühungen verschiedener Steueroasen, eine Quellenbesteuerung von Kapitaleinkommen unter Wahrung der Anonymität der Steuerhinterzieher als alternatives Instrument im Kampf gegen Steuerhinterziehung zu etablieren, ein Ende bereitet. Insbesondere die Schweiz hatte verschiedenen Ländern, darunter Deutschland, bilaterale Steuerabkommen auf der Basis dieses Instruments angeboten. In Deutschland scheiterte das Abkommen am Veto des Bundesrats. Darüber hinaus gab FATCA auch den Impuls für eine grundlegende Reform des global geltenden und von der OECD entwickelten Standards zum Informationsaustausch. Im Oktober 2014 wurde ein multilaterales Abkommen zur administrativen Kooperation in Steuersachen abgeschlossen, das den automatischen Informationstausch vorsieht (näher dazu: Rixen 2014). ${ }^{4}$

Schließlich hat FATCA maßgeblich dazu beigetragen, eine Verschärfung der EU-Zinsrichtlinie politisch durchsetzbar zu machen. Seit 2012 wurde auf EU-Ebene über eine Revision und Verschärfung der Zinsrichtlinie verhandelt. Es ging darum, den anonymen Quellenabzug für die EU-Mitglieder Österreich und Luxemburg sowie die ebenfalls von der Zinsrichtlinie erfasste Schweiz durch einen Wechsel zum automatischen Informationsaustausch zu beenden. Außerdem wurde der Einkommensbegriff erweitert. In Zukunft werden auch Dividenden, Investmentfonds, innovative Finanzinstrumente und Stiftungen erfasst. Nach anfänglichem Blockieren erklärte Luxemburgs Finanzminister, dass es kaum möglich sein werde, den europäischen Partnern zu verweigern, was man den Amerikanern mit FATCA ebenfalls gewähre. Die deutlich verbesserte Zinsrichtlinie, in der alle Staaten am automatischen Informationstausch teilnehmen, wurde im März 2014 verabschiedet (Hakelberg 2015).

Die persönliche Einkommensteuer muss im Land des Wohnsitzes gezahlt werden. Da natürliche Personen selten aus steuerlichen Gründen den Wohnsitz wechseln, reicht es zur Unterbindung der illegalen Steuerhinterziehung aus, wenn die Steueroasen zur Transparenz verpflichtet werden können. $\mathrm{Zu}$ erreichen ist dies durch einen automatischen Informationsaustausch und andere Formen der administrativen Kooperation zwischen Steuerbehörden. Eine Angleichung der Einkommensteuersätze und/oder Bemessungsgrundlagen ist wegen der geringeren Mobilität von natürlichen Personen jedoch nicht nötig. Die nationale Steuersouveränität wird nur in einem geringen Maße berührt. Diese Tatsache ist ein Grund dafür, warum man auf diesem Feld in den letzten Jahren wichtige Fortschritte erzielen konnte, wenn auch noch Lücken in der Durchsetzung bleiben.

\subsection{Der Kampf gegen Unternehmens- steuerwettbewerb}

Parallel zu einer Initiative der OECD (1998) begann die EU mit der Definition unfairer Steuerpraktiken im Bereich der Unternehmensbesteuerung. Im Jahr 1997 verabschiedete der Europäische Rat einen Verhaltenskodex gegen schädlichen Steuerwettbewerb, mit dem die Mitgliedstaaten eine Selbstverpflichtung eingingen, auf Steuerpraktiken zu verzichten, die ausländischen Unternehmen steuerliche Vorteile einräumten, die Inländern nicht offenstanden. Die 66 ursprünglich in den alten Mitgliedsländern und die weiteren 30 in den Beitrittsländern identifizierten schädlichen Steuerregime sind inzwischen weitgehend abgeschafft. Obwohl der Kodex nicht bindend ist, entwickelt er eine nicht zu unterschätzende Wirkung. Seine Einhaltung wurde zur Bedingung für die Aufnahme der Neumitglieder im Jahre 2005 gemacht. Außerdem wendet die Kommission die Prinzipien im Rahmen der Beihilferegeln (Art. 107 AEUV) an und erhöht dadurch ihre Bindungswirkung. Auch vier der im Rahmen von LuxLeaks bekannt gewordenen verbindlichen Steuerauskünfte (tax rulings) - die Fälle Starbucks, Apple, Fiat und Amazon - wurden bereits seit 2013 von der Kommission dahin gehend geprüft, ob sie mit dem Beihilferecht vereinbar waren. Im Oktober 2015 entschied die Kommission, dass die Niederlande einer Kaffeerösterei der Starbucks-Gruppe und Luxemburg einer Finanzierungsgesellschaft von Fiat selektive Steuervorteile eingeräumt haben und diese nun zurücknehmen sowie Steuernachzahlungen bis zu 30 Mio. € eintreiben müssen. In den Fällen Apple und Amazon steht eine Entscheidung noch aus.

Allerdings hat die an sich zu begrüßende Bekämpfung diskriminierender (Unternehmen mit Sitz im Ausland bevorzugende) Besteuerungsregeln auch zweifelhafte Nebenwirkungen: Da sie nun nicht mehr zugunsten ausländischer Unternehmen diskriminieren durften, boten manche EUStaaten daraufhin allen Steuerzahlern extrem niedrige Steuersätze an (Genschel et al. 2008). ${ }^{5}$

3 Die USA haben nicht teilnehmenden Finanzinstituten damit gedroht, von allen Zahlungen, die aus den USA an diese gehen, einen Quellenabzug in Höhe von $30 \%$ vorzunehmen. Wegen der großen Bedeutung des US-Marktes wäre dies für die betroffenen Banken ein beträchtlicher Wettbewerbsnachteil.

4 Die USA haben dieses Abkommen, obwohl sie die Durchsetzung des automatischen Informationstausches maßgeblich befördert haben, nicht unterzeichnet. Sie werden also in Zukunft von den Informationen anderer Staaten profitieren, können sich aber selbst als Steueroase für ausländische Anleger positionieren.

5 Irland beispielsweise schaffte nach Verabschiedung des Verhaltenskodizes seine Sondersteuersätze von $10 \%$ ab, senkte gleichzeitig aber den generellen Körperschaftsteuersatz auf $12,5 \%$. 
Außerdem hat sich die EU-Kommission darum bemüht, die administrative Kooperation zwischen den Mitgliedstaaten zu verbessern und sie zur Schaffung von Transparenz und Informationsaustausch anzuhalten. Hierzu hat man eine Reihe von Empfehlungen vorgelegt (z. B. Europäische Kommission 2009, 2012), aber auch einzelne Richtlinien im Rat (insbesondere Richtlinie 2011/16/EU) verabschieden können. Diese Bemühungen betreffen prinzipiell alle direkten Steuern. Hierbei geht es darum, Standards für die administrative Kooperation festzulegen, um sicherzustellen, dass sich kein Mitgliedstaat der Zusammenarbeit verweigern kann. Allerdings wird die Richtlinie nur unzureichend umgesetzt. Nach Ansicht vieler wäre beispielsweise Luxemburg zur Meldung seiner kontroversen „rulings“ verpflichtet gewesen, unterließ dies aber. Geplant ist für die Zukunft, einen automatischen Informationsaustausch über die verbindlichen Steuerauskünfte festzulegen. ${ }^{6}$ Außerdem gibt es einen Untersuchungsausschuss, in dem die luxemburgischen Praktiken einer genaueren Analyse unterzogen werden. Es bleibt abzuwarten, welche Konsequenzen daraus gezogen werden.

Insgesamt lässt sich sagen, dass man im Kampf gegen persönliche Steuerhinterziehung in den letzten Jahren wichtige Erfolge erzielt hat, während im Bereich der Unternehmenssteuern zu wenig erreicht wurde. Zwar hat man Fortschritte gegen den präferentiellen Steuerwettbewerb erzielen können, den generalisierten Wettbewerb hat man damit aber eher verschärft. Insbesondere ist zu bezweifeln, dass sich das Problem überhaupt durch administrative Kooperation lösen lässt. Anders als natürliche Personen sind Unternehmen und vor allem ihre Buchgewinne international mobil. Nur durch eine parametrische Koordinierung, also eine zumindest teilweise Harmonisierung von Bemessungsgrundlagen und/oder Steuersätzen, würde der Anreiz für die Unternehmen schwinden, Steuerdifferentiale auszunutzen. Während ein automatischer Informationsaustausch und die wechselseitige Information und Amtshilfe dem Problem der Steuerhinterziehung wirksam Abhilfe schaffen können, reicht sie im Kampf gegen den Unternehmenssteuerwettbewerb nicht aus. Denn Transparenz und Informationstausch ändern ja noch nichts daran, dass die Staaten frei darin sind, sich gegenseitig mit niedrigen Steuersätzen zu unterbieten (solange sie nicht-diskriminierend sind).

\section{Eine politische Alternative}

Der Steuerwettbewerb um die Unternehmenssteuern kann nur beschränkt werden, wenn der Einstieg in eine parametrische Kooperation gelingt. Eine Möglichkeit, dies zu erreichen, wäre die Einführung einer gemeinsamen konsolidierten Körperschaftsteuerbemessungsgrundlage (GKKB). Durch eine GKKB können die Möglichkeiten zur Gewinnverlagerung, die sich, wie in Abschnitt 2 skizziert, durch die national getrennte Gewinnermittlung ergeben, ausgeschaltet werden. Bei einer konsolidierten Gewinnermittlung mit Formelzerlegung wird der Gewinn (und Verlust) eines Unternehmens nicht national getrennt erhoben, sondern es wird ein Gesamtergebnis für das gesamte MNU (genauer: für die „Körperschaft“) ermittelt. Dies bedeutet, dass die Unternehmen Gewinne und Verluste über Ländergrenzen hinweg verrechnen können („konsolidiert“). Hierzu muss es europäische Regeln zur steuerlichen Gewinnermittlung geben, auf die sich alle Mitgliedsländer einigen können („gemeinsam“). Nachdem der steuerbare Gesamtertrag („Bemessungsgrundlage“) festgestellt ist, wird er entsprechend einer Verteilungsformel, die sich möglichst an realen ökonomischen Faktoren orientieren sollte, auf die beteiligten Länder aufgeteilt (Formelallokation). Wenn die Formel adäquat gewählt wird, fallen die wichtigsten Möglichkeiten zur Gewinnverlagerung für die Unternehmen weg. Artifizielle Konstrukte verlieren ihre Bedeutung, wenn sich die Zuteilung des Gesamtgewinns auf die beteiligten Länder an realen ökonomischen Größen orientiert. Bewährte Formeln greifen auf die drei Faktoren Lohnsumme, Vermögen und Umsatz der jeweiligen Zweigstelle zurück.

Allerdings birgt die Einführung einer GKKB die Gefahr, dass sich der reale Steuerwettbewerb verschärft. Wenn die Unternehmen nicht mehr mittels Gewinnverlagerung Steuervorteile realisieren können, dann steigt, wie oben erläutert, wahrscheinlich ihre Bereitschaft, reale Produktionsstandorte zu verlagern. Aus diesem Grund ist es notwendig, die GKKB mit einem Mindeststeuersatz zu versehen, den kein Mitgliedstaat unterbieten darf.

Die Einführung einer GKKB steht in der EU bereits seit Längerem auf der Agenda. Seit Beginn der 2000er Jahre verfolgt die Kommission die Absicht, eine GKKB einzuführen. Nachdem sie einen Richtlinienentwurf im Jahr 2008 wegen negativer Signale vonseiten einiger Mitgliedstaaten gar nicht erst einbrachte, hat sie im Jahr 2011 einen entschärften Richtlinienentwurf vorgelegt, der aber bisher ebenfalls keine Mehrheiten im Rat fand. ${ }^{7}$ Der Entwurf bietet zwar einen Ansatzpunkt zu einer weitergehenden Harmonisierung, weist jedoch erhebliche Mängel auf: Erstens würden die Unternehmen wählen können zwischen dem bisherigen System und der GKKB. Dies würde die Gestaltungsmöglichkeiten eher erhöhen als begrenzen. Zweitens ist kein Mindeststeuersatz festgelegt worden (zu diesen und weiteren Mängeln des Entwurfs siehe Rixen/Uhl 2011).

6 Damit wären die „rulings" selbst freilich noch nicht aufgegeben, aber es würde sich für die anderen Mitgliedstaaten eine Möglichkeit ergeben, mit steuerlichen Abwehrgesetzen darauf zu reagieren.

7 Zu den politischen und technischen Schwierigkeiten, die sich mit einer GKKB verbinden, vgl. z. B. Uhl/Rixen (2007) und Fuest (2008). 


\section{Ausblick}

In diesem Beitrag habe ich argumentiert, dass der Steuerwettbewerb die Ungleichheiten in und zwischen den EU-Mitgliedsländern erhöht und deshalb mit den Zielen eines sozialen Europas nicht vereinbar ist. Die EU sollte deshalb nicht nur den virtuellen und präferentiellen Steuerwettbewerb, der häufig als ,unfair“ bezeichnet wird, regulieren, sondern auch den generalisierten Wettbewerb. Eine Möglichkeit dazu böte die GKKB mit Mindeststeuersatz. Diese bietet die Aussicht auf eine Rückgewinnung der tatsächlichen steuerpolitischen Gestaltungsfähigkeit durch die Nationalstaaten. Sie lässt den Staaten Spielraum bei der Festsetzung der Steuerpolitik, bietet aber ein Maß an parametrischer Kooperation, das sicherstellt, dass sie die für die Verwirklichung eines sozialen Europas so wichtigen Verteilungsziele auch tatsächlich erreichen können und diese nicht, wie bisher, im Steuerwettbewerb unter die Räder geraten.

Angesichts der Tatsache, dass sich wichtige EU-Akteure und viele Regierungen lediglich gegen den präferentiellen und virtuellen Steuerwettbewerb wenden, den generalisierten und vermeintlich „fairen“ Steuerwettbewerb um reale Investitionen aber begrüßen (siehe z.B. Europäische Kommission 2011), stehen die Aussichten für eine solche Reform derzeit schlecht. Das ändert aber nichts daran, dass sie notwendig ist. Es ist an der Zeit, dass die politischen Kräfte, die sich ein soziales Europa auf die Fahnen geschrieben haben, die Wichtigkeit einer gemeinsamen europäischen Steuerpolitik erkennen.

\section{LITERATUR}

Clausing, K. A. (2016): The nature and practice of capital tax competition, in: Dietsch, P./Rixen, T. (Hrsg.): Global tax governance - what is wrong with it and how to fix It, Colchester (im Erscheinen)

De Mooij, R. A./Ederveen, S. (2008): Corporate tax elasticities: a reader's guide to empirical findings, in: Oxford Review of Economic Policy 24 (4), S. 680-697 Elschner, C./Vanborren, W. (2009): Corporate effective tax rates in an enlarged European Union, European Commission Taxation Paper (14), Luxembourg Europäische Kommission (2009): Förderung des verantwortungsvollen Handelns im Steuerbereich, KOM(2009) 201, http://ec.europa.eu/taxation_customs/ resources/documents/common/whats_new/COM\%282009\%29201_de.pdf (letzter Zugriff: 06.11.2015)

Europäische Kommission (2011): Staatliche Beihilfen: Kommission prüft Verrechnungspreisvereinbarungen im Rahmen der Besteuerung von Apple (Irland), Starbucks (Niederlande) und Fiat Finance and Trade (Luxemburg), Pressemitteilungen vom 11. Juni, http://europa.eu/rapid/press-release IP-14-663_de.htm

Europäische Kommission (2012): Mitteilung der Kommission an das Europäische Parlament und den Rat: Aktionsplan zur Verstärkung der Bekämpfung von Steuerbetrug und Steuerhinterziehung, http://ec.europa.eu/taxation_customs/ resources/documents/taxation/tax_fraud_evasion/com_2012_722_de.pdf (letzter Aufruf: 06.11.2015)

Eurostat (2008): Taxation trends in the European Union. Data for the EU member states and Norway, Eurostat Statistical books, Luxemburg

Eurostat (2014): Taxation trends in the European Union. Data for the EU member states, Iceland and Norway, Eurostat Statistical books, Luxemburg

Fuest, C. (2008): The European Commission's proposal for a common consolidated corporate tax base, in: Oxford Review of Economic Policy 24 (4), S. $720-739$

Ganghof, S./Genschel, P. (2008): Taxation and democracy in the EU, in: Journal of European Public Policy 15 (1), S. 58-77

Genschel, P./Jachtenfuchs, M. (2011): How the European Union constrains the state: multilevel governance of taxation, in: European Journal of Political Research 50 (3), S. 293-314
Genschel, P./Kemmerling, A./Seils, E. (2011): Accelerating downhill: how the EU shapes corporate tax competition in the Single Market, in: Journal of Common Market Studies 49 (1), S. 1-22

Genschel, P./Rixen, T./Uhl, S. (2008): Die Ursachen des europäischen Steuerwettbewerbs, in: Politische Vierteljahresschrift Sonderheft 40/2008, S. 297-320 Godar, S./Paetz, C./Truger, A. (2015): Spielräume für eine gerechtere Steuerpolitik in den OECD-Ländern, in: WSI-Mitteilungen 68 (4), S. 263-271,

http://www.boeckler.de/wsi-mitteilungen_54287_54301.htm

Hakelberg, L. (2015): The power politics of international tax cooperation: Luxembourg, Austria and the automatic exchange of information, in: Journal of European Public Policy 22 (3), S. 409-428

OECD (Organisation for Economic Co-operation and Development) (1998): Harmful tax competition. An emerging global issue, Paris

Palan, R./Murphy, R./Chavagneux, C. (2010): Tax havens. How globalization really works, Ithaca

Piketty, T. (2014): Das Kapital im 21. Jahrhundert, München

Rixen, T. (2013): Der Kampf gegen Steuerwettbewerb und Steuerflucht: Entwicklungslinien der internationalen Steuerpolitik, in: DIW Vierteljahreshefte zur Wirtschaftsforschung (Höhere "Reichensteuern“: Möglichkeiten und Grenzen) 82 (1), S. $61-75$

Rixen, T. (2014): Internationale Steuerflucht und schädlicher Steuerwettbewerb, in: politische bildung 47 (4), S. 34-54

Rixen, T./Schwarz, P. (2012): How effective is the European Union's Savings Tax Directive? Evidence from four EU member states, in: Journal of Common Market Studies 50 (1), S. 151-168

Rixen, T./Uhl, S. (2011): Unternehmensbesteuerung europäisch harmonisieren! Was zur Eindämmung des Steuerwettbewerbs in der EU nötig ist, Gutachten im Auftrag der Friedrich-Ebert-Stiftung, http://library.fes.de/pdf-files/id/ ipa/08462.pdf (letzter Zugriff 05.11.2015)

Sinn, H.-W. (1997): Das Selektionsprinzip und der Systemwettbewerb, in: Oberhauser, A. (Hrsg.): Fiskalföderalismus in Europa, Schriften des Vereins für Sozialpolitik, Berlin, S. 9-60

Tiebout, C. M. (1956): A pure theory of local expenditures, in: Journal of Political Economy 64 (5), S. 416-424

Uhl, S./Rixen, T. (2007): Unternehmensbesteuerung europäisch gestalten mitgliedstaatliche Handlungsspielräume gewinnen. Gutachten für die FriedrichEbert-Stiftung, http://library.fes.de/pdf-files/id/04575.pdf (letzter Zugriff 05.11 2015) Wasserfallen, F. (2014): Political and economic integration in the EU: the case of failed tax harmonization, in: Journal of Common Market Studies 52 (2), S. $420-435$

Winner, H. (2005): Has tax competition emerged in OECD Countries? Evidence from panel data, in: International Tax and Public Finance 12 (5), S. 667-687 Zucman, G. (2014): Steueroasen. Wo der Wohlstand der Nationen versteckt wird, Berlin

\section{AUTOR}

THOMAS RIXEN, Prof. Dr., lehrt Politikwissenschaft, insbes. international vergleichende Politikfeldanalyse an der Universität Bamberg. Arbeitsschwerpunkte: Internationale und Vergleichende Politische Ökonomie, Institutionalismus und Steuer-, Finanz- und Sozialpolitik.

thomas.rixen@uni-bamberg.de 